\title{
(2) OPEN ACCESS \\ What characterises work and workplaces that retain their employees following acquired brain injury? Systematic review
}

\author{
Daniele Evelin Alves (1) , Wendy Nilsen, ${ }^{1}$ Silje Christine Reistad Fure, 2,3 \\ Heidi Enehaug, ${ }_{1}^{1}$ Emilie Isager Howe, ${ }_{1}^{4,5}$ Marianne Løvstad, ${ }^{6,7}$ Louisa Fink, ${ }^{8}$ \\ Nada Andelic, ${ }^{4,9}$ Øystein Spjelkavik'
}

\begin{abstract}
- Additional material is published online only. To view, please visit the journal online (http://dx.doi.org/10.1136/ oemed-2019-106102).
\end{abstract}

For numbered affiliations see end of article.

\section{Correspondence to}

Dr Daniele Evelin Alves, Work Research Institute, Oslo Metropolitan University, Oslo, Akershus, Norway; daniele.alves@oslomet.no

Received 22 July 2019 Revised 11 December 2019 Accepted 14 December 2019

Published Online First 6 January 2020

\section{Check for updates}

(c) Author(s) (or their employer(s)) 2020. Re-use permitted under CC BY-NC. No commercial re-use. See rights and permissions. Published by BMJ.

To cite: Alves $D E$,

Nilsen W, Fure SCR, et al

Occup Environ Med

2020:77:122-130.

\begin{abstract}
Objectives The objective of this study was to conduct a systematic review assessing workplace factors related to work retention (or return to work) in employees with acquired brain injury (ABI). Additionally, we aimed to synthesise the evidence and state of knowledge on this subject.
\end{abstract}

Methods A database search was performed in nine relevant electronic databases. Inclusion criteria were quantitative peer-reviewed publications empirically investigating the relationship between work/workplace factors and work retention in employees following $A B I$. The methodological quality was determined by Effective Public Health Practice Project scoring, and evidence was synthesised narratively.

Results Thirteen studies were included. We found moderate evidence for a negative relationship between manual work and work retention. We also found limited evidence for a U-shaped relationship between workload and complete work retention at 6 months and no relationship at 12 months; a positive relationship between managers, compared with non-managers, and faster work retention; a positive relationship between large enterprise size defined as $\geq 250$ employees, and no relationship between large enterprise size, defined as $\geq 1000$ employees, and work retention.

Conclusion Relative to individual factors, there is little evidence on specific workplace factors' relationship to work retention among employees with $\mathrm{ABI}$. For most workplace factors, there were too few high-quality studies to designate evidence as more than limited or insufficient. Future studies should replicate rigorous studies of well-defined modifiable workplace factors related to work retention.

PROSPERO registration number CRD42018082201.

\section{INTRODUCTION}

Acquired brain injury (ABI) is defined as damage to the brain caused by traumatic or non-traumatic injury. ${ }^{1}$ Traumatic brain injury (TBI) refers to non-congenital and nondegenerative damage to the brain that is inflicted by an external force. Non-TBI, on the other hand, refers to a range of neurological illnesses (eg, stroke, tumours, brain infections and hypoxia). Although the distinction between TBI and non-TBI is essential for defining early patient management programmes, research suggests that patients suffering from any of the two types of brain injuries report similar challenges

\section{Key messages}

What is already known about this subject?

- Most single studies and reviews on factors related to return to work for employees with acquired brain injury (ABI) focus on unmodifiable, individual factors such as age, gender and medical characteristics. Although important, individual factors alone cannot translate into guidelines for adaptations at workplaces in order to increase work retention.

\section{What are the new findings?}

- This is the first review to focus and systematically summarise evidence on workplace factors associated with work retention after $A B I$. The review identified few studies and little sound evidence regarding the specific workplace factors of importance. We found moderate evidence that manual work was negatively associated with work retention, while there was limited and equivocal evidence for the relationship between managerial role, workload and enterprise size, and work retention.

How might this impact on policy or clinical practice in the foreseeable future?

- Future studies should examine specific modifiable workplace factors by using rigorous designs and well-defined and comparable definitions of work retention. This is necessary to enable the transfer of evidence synthesis into clinical and policy impact. Until then, we suggest that workplaces pay special attention to manual workers after $A B I$, researchers replicate the strong-quality and moderatequality studies identified in this review to increase evidence base, and that employers temporarily rely on review findings from other diagnostic groups.

when returning back to work. Some of the similar and important barriers are cognitive disabilities, emotional problems and fatigue. ${ }^{1}$ Further, individuals with $\mathrm{ABI}$ tend to shift focus from work to their social networks, struggling to return to a state of normality at the workplace, and perceiving a threat to their competence and work identity. These similarities justify our approach to combine both types 
of brain injuries when studying work retention, as other studies have done.

Both traumatic and non-traumatic ABI can lead to permanent or temporary disabilities that may limit individuals in their daily activities at home and at work. The prevalence of long-term disability resulting from TBI has been estimated at 3.32 million $^{2}$ to 5.3 million $^{3}$ in the USA, and 7.7 million people in the European Union. ${ }^{4}$ For stroke, the estimated global burden included more than eight million survivors in 2016. Hence, ABI imposes a heavy societal burden due to the healthcare and social and vocational support costs, as well as disability pensions involved. ${ }^{67}$

Approximately $70 \%$ of patients with ABI are of working age. ${ }^{2}$ Studies show that both types of ABI negatively affect work retention or return to work (RTW). People with ABI are employed less often, and when employed, they frequently experience difficulties in meeting the physical, cognitive or psychosocial demands of the workplace. ${ }^{89}$ To improve work participation rates after $\mathrm{ABI}$, we need to increase the understanding of what characterises workplaces that retain their employees following ABI.

The complexity of the relationship between brain injury and RTW has been underlined in different reviews. ${ }^{9-12}$ Shwarz et al ${ }^{10}$ pointed out four types of factors predicting RTW: individual, workplace, rehabilitation and their interaction. The authors proposed that a model predicting RTW after stroke should include diverse factors and stakeholders, such as employees, employers, vocational therapists and families.

Most studies investigating work retention after $\mathrm{ABI}$ include only individual factors. ${ }^{13-22}$ Well-established individual factors related to increased work retention are lower injury severity, higher education and male gender. Although undoubtedly important, individual factors alone cannot translate into guidelines for adaptations at workplaces to increase work retention. Compared with individual factors, specific workplace factors are relatively scarce in original studies. Studies that do include workplace factors tend to include them across vast patient populations (all types of injuries), ${ }^{23}$ in general terms (eg, as one of many overarching factors ${ }^{24}$ or parts of a complex intervention), 8202526 and lack specificity as to which particular workplace factors are effective. For instance, Donker-Cools et al found strong evidence of the effectiveness of work-directed interventions combined with education or coaching, ${ }^{8}$ while other general reviews report that interventions were similarly effective. ${ }^{202526}$ Although informative, reviews including general workplace factors do not identify specific workplace factors associated with work retention. In addition to the skill-advancing programme that aids RTW, there is a need to develop programmes that address the struggles faced by former patients with ABI to retain work. A deeper understanding of the workplace factors that facilitate work retention among former patients with $\mathrm{ABI}$ is thus necessary for the development of such programmes. However, to the best of our knowledge, there are no systematic reviews examining the relationship between workplace factors and work retention after ABI. The objective of the current study was thus to fill this gap with a comprehensive systematic review to assess the evidence and state of knowledge regarding workplace factors' role in work retention after ABI.

\section{METHODS}

A systematic search using keywords and medical subject heading terms was conducted in February 2018, in the electronic databases Ovid, Web of Science, Scopus, Embase, PsyINFO, SocIndex, Cinahl, Academic search premier, Cochrane Library and Norart. We combined two clusters of keywords and subject headings related to (1) the participants (eg, brain injury, acquired, traumatic and stroke) and (2) the outcome (eg, work/ job/employment maintenance, tenure, retention, hours and RTW). This combination of search words enabled any workplace factor (including terminology from other disciplines) to be identified during screening. We collaborated closely with librarians who specialised in systematic reviews and tried out different words and combinations that enabled the identification of as many relevant workplace factors as possible (see appendix 1 for the complete search strategy for each database). At least two reviewers screened the abstracts independently of each other with the online tool Covidence. ${ }^{27}$ In cases of disagreement, the reviewers discussed until accordance was reached, or a third reviewer was involved. Relevant full texts were retrieved and read.

This systematic review is registered with International Prospective Register of Systematic Reviews (PROSPERO). ${ }^{28}$ Guidelines of the Preferred Reporting Items for Systematic Reviews and Meta-Analyses Protocols checklist ${ }^{29}$ and the 'PICO' approach were used. ${ }^{30}$ The review team was multidisciplinary and consisted of researchers within the field of occupational psychology, work sociology and neurorehabilitation. In the original protocol, the review included both quantitative and qualitative studies. To improve coherence when synthesising data, we split the original mixed-method review into two manuscripts: one including quantitative studies (the current review) and one including qualitative studies to be published later. Qualitative studies are characterised 'wrong study design' in the current review (see figure 1), and deviation from protocol is documented in PROSPERO.

\section{Inclusion and exclusion criteria}

Inclusion criteria were peer-reviewed publications quantitatively and empirically investigating the relationship between workplace factors and work retention/RTW in adult employees (ages 18-65 years) in paid employment at the time of ABI. The exposure of interest included all types of workplace factors. Examples of workplace factors were work accommodation, work adaption, flexible work hours, workplace size, interventions involving the workplace, and social support by coworkers and managers. Occupational role was included in the framework of workplace factors because it describes characteristics of the occupation that are related to work retention and can be modified by the workplace to adapt work for employees with brain injury. Individual factors not pertaining to the workplace (eg, personality, age, injury characteristics and medical variables) were excluded from this review. The outcome of interest was work retention (eg, maintenance, tenure and persistent RTW) operationalised in measure of time (eg, days and years) and dichotomous outcomes (eg, work participation 6 months after RTW: yes/no). Studies were included if the relationship between exposure and the outcome variables was tested for statistical significance. There was no publication year limit, as we expected to find few studies investigating the same specific workplace factor and aimed for a wide search. Language was restricted to English, Norwegian, Danish, Swedish, Spanish and Portuguese.

\section{Data extraction}

One reviewer extracted data from the included studies, which was double-checked by another reviewer. The data were extracted with a preagreed-upon standardised form covering (1) any workplace factor ('the exposure factor') investigated in relationship to the outcome; (2) operationalisation of the work 


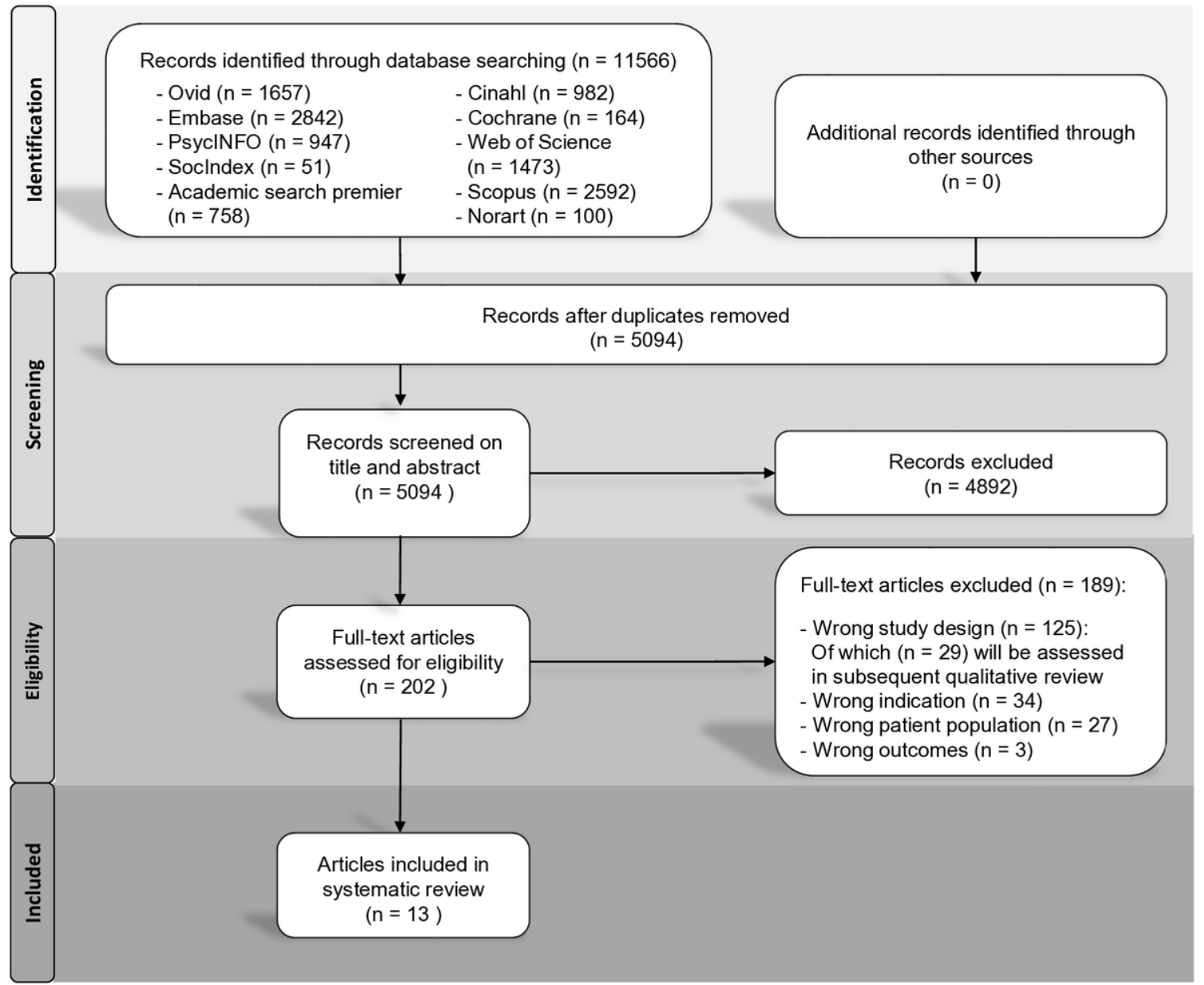

Figure 1 Flow chart of steps in systematic review.

retention and overlapping terms, such as job tenure or RTW ('the outcome'); (3) quantitative finding of the relationship between the exposure and the outcome; (4) the study characteristics (ie, sample size, study design and response rate); (5) country of origin; and (6) main findings. We grouped studies according to the type of workplace factors investigated in relation to work retention. Studies were then assessed for quality and synthesised for evidence within each workplace factor through a narrative synthesis (see further).

\section{Quality assessment}

The Effective Public Health Practice Project (EPHPP, appendix 2) checklist $^{31}$ was used to assess the quality of the included quantitative studies. Two independent reviewers (authors DEA and SCRF), assessed the quality of each quantitative study into strong, moderate or poor quality. Studies were rated in terms of different components (A-F, see further) and in terms of global ratings. Discrepancies were resolved by discussions or involving a third reviewer (WN). The following six components were evaluated: (A) selection bias, (B) study design, (C) confounders, (D) blinding, (E) data collection methods and (F) withdrawals and drop-outs. A global score was given by transforming each component rating into scores: strong $=3$, moderate $=2$, weak $=1$ or not applicable $=0$, and summing component ratings, yielding a total score ranging from 5 to 18 for each study. According to its global rating, each study was designated strong (no weak component ratings), moderate (no more than one weak component rating) or weak (more than one weak rating). ${ }^{31}$

\section{Evidence synthesis}

Finally, we synthesised findings for each specific workplace factors following each study's EPHPP global rating, and the systematic review evidence synthesis guide (appendix 3 ). ${ }^{32}$ The guide's synthesis algorithm ranges the level of evidence from strong to insufficient. ${ }^{32-34}$ Evidence is rated strong if three strong studies agree, or if more than three studies, three-fourths of the moderate and strong studies agree. Moderate evidence refers to two strong studies agreeing, or two moderate studies and one strong study agreeing (if more than three studies, more than two-thirds of the moderate and strong studies agree). Limited evidence refers to two moderate and/or strong studies agreeing (if more than two studies, more than one-half of the moderate and strong studies agree). Mixed evidence refers to moderate and strong studies showing contradictory results. Insufficient evidence refers to no strong studies, only one moderate study and/or any number of weak studies.

\section{RESULTS}

The search revealed 5094 records after removing duplicates. After screening abstracts, 202 titles matched the inclusion criteria and were read in full text. Thirteen studies examined statistical associations between workplace factors and work retention after $\mathrm{ABI}$ and were included after reading full-text articles $^{35-47}$ (see figure 1 for the full Preferred Reporting Items for Systematic Reviews and Meta-Analyses flowchart and reasons for exclusion). 
Table 1 Summary of studies included in the systematic analysis*

\begin{tabular}{lllll}
\hline Study & Sample (response rate) & Study design, follow-up time & Exposure(s) & Outcome(s) \\
\hline Autret et al (2015) & 76 French patients with ABl aged & Longitudinal, $>2$ years' follow-up & Blue collar versus white collar & RTW versus no RTW \\
& $\begin{array}{llll}18-60 \text { years from a rehabilitation } \\
\text { clinic followed up by an }\end{array}$ & & & \\
& occupational therapist (N/A) & & &
\end{tabular}

141 Indian patients with stroke medical centres (NR)

Bonneterre et al (2013)

\section{French TBI adult participants} enrolled in a programme for work reintegration ('SPASE' + ), wishing to RTW (N/A)

Longitudinal, 'short term' (2-3 years) and 'medium term' (ove 3 years)

2-year follow-up

de Koning et al (2017) 319 Dutch employees with mild TBI Longitudinal; 6 and 12 months at the emergency department (74\%) after injury

\begin{tabular}{|ll}
\hline Endo et al (2016) & $\begin{array}{l}382 \text { Japanese employees with } \\
\text { stroke (N/A) }\end{array}$ \\
\hline Hannerz et al (2011) & $\begin{array}{l}19903 \text { Danish employees with } \\
\text { stroke, aged 20-57 years (99.6\%) }\end{array}$
\end{tabular}

Hannerz et al (2012)

12106 Danish employees with stroke, aged $21-57$ years $(92 \%)$

Johnson (1987)

47 British employees with severe TBI (NR)
Cross-sectional, N/A aged 18-60 years from tertiary
Longitudinal, 2 years after stroke

Longitudinal, mean follow-

up time 3.5 years after injury (minimum of 2.5 years)
Longitudinal; 2, 4, 6 and 12 months after first sickness absence day

Longitudinal, 2 years after stroke
Manual labour versus business or professional labour (written/oral self-report)

RTW versus no RTW

1. Workplace support (present or absent). RTW 2-3 years after injury and

2. Occupational assistance (sporadic or regular).

1. Professional/managerial (eg, executive or managerial function) or skilled (eg, sales and administrative support) or manual labour (eg, machine operators and private household).

2. Workload in hours per week (40 hours/ week=full time).

1. Manual versus desk worker.

2. Manager versus non-manager.

3. Company size ( $\leq 999$ employees or $\geq 1000$ employees).

\section{Occupational class}

(DISC0-88 classification: (1) Legislators, senior officials and managers; (2)

technicians and associate professionals;

(3) workers in occupations that require basic skills; (4) workers in elementary occupations; (5) gainfully occupied people with unknown occupation)

Enterprise size (micro, 1-9 employees; small, Odds of returning to work 10-49; medium, 50-249; large, $\geq 250$ )

1. Normal work conditions (normal fulltime and part time).

2. Return under special conditions (easier work, testing of employees' capability, informal return, liaison with the rehab. unit, work training and workplace support).

$\begin{array}{ll}\text { Ntsiea et al (2015) } & \begin{array}{l}80 \text { South African patients with } \\ \text { stroke, aged 18-60year }(96.4 \%)\end{array} \\ \text { Saeki et al (1995) } & \begin{array}{l}183 \text { Japanese patients with stroke, } \\ \text { younger than 65 years (N/A) }\end{array}\end{array}$

Randomised controlled trial,

Workplace intervention tailored according 6 months after intervention (circa 8 months after injury)

to functional ability and workplace challenges

Longitudinal; 6,12 and 18 months White collar versus blue collar and up to approximately 3 years after hospital admission for injury

van Dongen et al (2018) 58 Dutch patients with $\mathrm{ABI}(87.9 \%)$ Longitudinal, follow-up 3-6years
after the vocational rehabilitation (around 4-7 years after injury)

Vestling et al (2003) 120 Swedish patients with stroke, younger than 60 years (N/A)

Longitudinal, minimum of 6 months poststroke (average of 2.7 years after injury)

Walker et al (2006)
Vocational rehabilitation programme with multidisciplinary assessment, stakeholder meetings (including family, employer and coworker), on-the job training and coaching White-collar and blue-collar dichotomised (yes/no) (Swedish socioeconomic classification)

Professional/managerial, skilled or manual labour (The International Standard Classification of Occupations) over 3 years after injury

Complete RTW (same work hours preinjury and postinjury) versus no/part RTW (less work hours postinjury than preinjury), 6 and 12 months after injury

(1) Full RTW or (2) resignation for the 365-day period following first day of sickness absence

Odds of returning to work

Continuous employment for at least 1 year after injury

RTW 6 months after intervention

RTW 1 month or more in active employment after stroke

\section{RTW}

(performing paid work: yes/no)

Work/no work

Competitive employment at 1 year postinjury (circa 8 months after injury)
1926 North American patients with TBI, aged $18-62$ years $(100 \%)$
Longitudinal, follow-up 1 year postinjury

Only exposures and outcomes tested for statistical significance are presented in table 1.

DISCO-88 is an acronym for Denmark's Standad Classification of Occupations.

*Sample refers to the number of participants according to response rate. N/A, for example, response rate for retrospective studies. NR, for example, response rate in registry cross-sectional study.

† SPASE is a non-English abbreviation of 'personalized service of accompaniment and follow-up to employment'.

$A B I$, acquired brain injury;N/A, not applicable; NR, not relevant; RTW, return to work; TBI, traumatic brain injury.

Table 1 summarises the study design and operationalisations of workplace factors and work retention in the 13 included studies. All studies were published in English between 1987 and 2017. The majority of the studies used a longitudinal design $(n=11)$; one used a randomised controlled trial (RCT) design $(n=1)^{43}$; and one cross-sectional design $(n=1){ }^{36}$ The duration of follow-up or time since injury varied from 6 months to 7 years. The studies gathered data from nine 
Table 2 Workplace factors related to work retention

\begin{tabular}{|c|c|c|c|c|}
\hline Workplace factors & Sample (n) & Country & Findings & Source \\
\hline Work accommodation & 47 , severe $\mathrm{TBI}$ & Great Britain & $\begin{array}{l}\text { Combination of work trials, assistance from coworker or fewer working hours } \\
\text { during a period after RTW related to continuous employment for at least } 1 \text { year } \\
\text { since injury }(p=0.05) \text {. }\end{array}$ & Johnson (1987) \\
\hline $\begin{array}{l}\text { Workload (hours/week) } \\
\text { ( } 40 \text { hours/week=full time) }\end{array}$ & 319, mild TBI & The Netherlands & $\begin{array}{l}\text { Work load related to complete RTW after } 6 \text { months in a U-shaped curve: less than } \\
25 \text { hours a week related to highest odds of RTW, } 32 \text { hours a week related to lowest } \\
\text { odds of complete RTW, followed by increased odds with increasing work load (OR } \\
1.25,95 \% \mathrm{Cl} 0.71 \text { to } 2.20) \text {. Workload not related to complete RTW } 12 \text { months after } \\
\text { injury }\end{array}$ & $\begin{array}{l}\text { de Koning et al } \\
\text { (2017) }\end{array}$ \\
\hline
\end{tabular}

Regularity of occupational assistance (sporadic vs regular)

Workplace intervention tailored according to functional ability and workplace challenges Vocational rehabilitation programme with multidisciplinary assessment, stakeholder meetings (including family, employer and coworker), on-the-job training and coaching Workplace support (present vs not present)

Blue collar ('manual' activity) vs white collar ('intellectual' activity)

Manual, business or professional labour*

Professional/managerial (eg, executive or managerial function) or skilled (eg, sales and administrative support) or manual labour (eg, machine operators and private household)*

Manual versus desk worker

manager versus non-manager*

Occupational class (DISCO-88 classification:

(1) legislators, senior officials and managers;

(2) technicians and associate professionals;

(3) workers in occupations that require basic

skills; (4) workers in elementary occupations;

(5) gainfully occupied people with unknown occupation)*

White-collar versus blue-collar occupations

White-collar, blue-collar and selfemployed dichotomised (yes/no) (Swedish socioeconomic classification)

Professional/managerial, skilled or manual labour (The International Standard

Classification of Occupations)*

Enterprise size (micro, 1-9 employees;

small, 10-49; medium, 50-249; large, $\geq 250$

employees)

Enterprise size ( $\leq 999$ or $\geq 1000$ employees)

$\begin{array}{ll}\text { 100, } \mathrm{TBI} & \text { France } \\ \text { 80, stroke } & \text { South Africa } \\ 58, \mathrm{ABI} & \text { The Netherlands } \\ 100, \mathrm{TBI} & \text { France } \\ 76, \mathrm{ABI} & \text { France } \\ 141, \text { stroke } & \text { India }\end{array}$

Regular versus sporadic occupational assistance: threefold increase in RTW

319, mild TBI The Netherlands

382, stroke Japan

19903, stroke Denmark

$\begin{array}{ll}\text { 183, stroke Japan } & \\ \text { 120, stroke } & \text { Sweden } \\ 1926, \mathrm{TBI} & \text { USA }\end{array}$

12106, stroke Denmark

382, stroke Japan $2-3$ years after injury $\left(\chi^{2}=3.73, p<0.05\right)$, no significant increase after $4-8$ years

Intervention group 5.2 times greater odds of returning to work at 6 months than control group

Average increase of 5.3 hours of work per week at long-term (circa 4-7years postinjury) compared with immediately after (around 1 year after injury, $p<0.01$ ) the intervention. On average, employees worked 5.9 hours of work per week less than before the injury $(p<0.01)$; no control group

Support at the workplace related to RTW 2-3years after injury (adjusted OR 15.13, $95 \% \mathrm{Cl} 3.17$ to 61.74 ) and $4-8$ years (adjusted OR $6.47,95 \% \mathrm{Cl} 1.96$ to 21.34 )

Blue-collar and white-collar workers demonstrated circa the same rate of RTW (blue collar 28/49=57\%, white collar $15 / 27=55 \%$; $p>0.99$ )

Higher RTW in professional and business jobs (adjusted OR $3.02,95 \% \mathrm{Cl} 1.44$ to 6.34) compared with manual jobs

Manual and professional (compared with skilled) labour related to the lowest odd of complete RTW after 6 months (both OR 0.38), occupational status not related to complete RTW after 12 months

Manual workers had a longer time to resignation than non-manual workers ( $\mathrm{HR}=0.24,95 \% \mathrm{Cl} 0.07$ to 0.80 ). Managers had shorter time to full RTW than non-managers 1 year after the first day of sick leave postinjury ( $H R=1.68,95 \% \mathrm{Cl}$ 1.02 to 2.78$)$. Controlled for age and sex in both models, and for desk worker/nondesk-worker in second model

Lower odds of RTW among workers in elementary occupations (refrence group) than those with occupations requiring basic skills 1.50 (95\% Cl 1.38 to 1.64), technicians and associated professionals $2.33(95 \% \mathrm{Cl} 2.05$ to 2.65$)$, and professionals $3.04(95 \% \mathrm{Cl} 2.70$ to 3.43$)$

Higher adjusted OR of successful RTW among white-collar compared with bluecollar workers (OR 5.16, $\mathrm{p}<0.05)^{*}$

Higher OR of RTW for white-collar workers (compared with others) was 2.99 $(p<0.063)$, ${ }^{*}$ and non-significant for blue-collar workers $(0.59, p=0.83) 6$ months postinjury. Self-employed was not analysed further after non-significant relationship with RTW on preliminary analysis

Higher odds of RTW for professional/managerial workers (OR 2.959, $\mathrm{p}<0.05)$ and skilled workers (OR 1.536, $\mathrm{p}<0.05)$ compared with manual workers. ORs adjusted for age, gender, education and severity are 3.155 and 1.706 , respectively)

Large enterprise ( $\geq 250$ employees) size related to RTW after 2 years $(p=0.034)$. Each increase in enterprise size category was followed by an increase in the odds of RTW.

(2013)

Ntsiea et al (2015)

van Dongen et al

(2018)

Bonneterre et al

(2013)

Autret et al (2015)

Bonner et al

(2016)

Endo et al (2016)

Hannerz et al

(2011)

Enterprise size unrelated to RTW and resignation 1 year after first sickness absence Endo et al (2016) day when controlled for age and sex

*Studies assessing manual occupational role included in evidence synthesis (strong or moderate). Sample refers to he number of participants according to the response rate. Only exposures and outcomes fulfilling inclusion criteria and tested for statistical significance are presented.

$A B I$, acquired brain injury; RTW, return to work; TBI, traumatic brain injury

countries covering four continents: Denmark $(n=2), 4041$ France $(n=2),{ }^{35} 37$ Japan $(n=2),{ }^{39} 44$ Netherlands $(n=2),{ }^{38} 45$ Great Britain $(n=1),{ }^{42}$ India $(n=1),{ }^{36}$ South Africa $(n=1),{ }^{43}$ Sweden $(n=1)^{46}$ and the USA $(n=1) .^{47}$

Sample sizes ranged from $47^{42}$ to $19903 .^{41}$ The majority of the studies examined stroke $(n=7),{ }^{36} 39-41434446$ followed by TBI $(n=4),{ }^{37} 384247$ while two studies investigated ABI of mixed aetiologies. ${ }^{35} 45$ Among studies collecting selfreport data $(n=10),{ }^{35-38} 42-47$ only data from employees were collected, while none of the studies included self-reported data from employers or occupational workers/vocational staff. Three studies included national registry data, ${ }^{40} 4147$ and nine studies collected data from clinical, occupational or hospital registries. ${ }^{35}$ 37-39 42-46 The included studies examined a total of 16 workplace factors and work retention after ABI (table 2).

Two studies assessed work adaptations with onsite assistance (assistance found within the workplace, eg, coworkers, managers and human resources). ${ }^{38} 42$ Three studies investigated work adaptations with external assistance (ie, assistance from outside the workplace, eg, therapist and employment counselor), ${ }^{37} 4345$ while only one study specifically investigated workplace social support. ${ }^{37}$ The majority of the studies $(n=8)$ were concerned with occupational role, ${ }^{3536383941444647}$ and two studies investigated enterprise size. ${ }^{3940}$ 
Table 3 Quality assessment of studies based on Effective Public Health Practice Project criteria: total score and quality according to global and component ratings*

\begin{tabular}{|c|c|c|c|c|c|c|c|c|}
\hline $\begin{array}{l}\text { Source, } \\
\text { country }\end{array}$ & $\begin{array}{l}\text { Total score } \\
(4-18)\end{array}$ & Global rating & Selection bias & Study design & $\begin{array}{l}\text { Control of } \\
\text { confounders }\end{array}$ & Blinding & $\begin{array}{l}\text { Data } \\
\text { collection } \\
\text { methods }\end{array}$ & $\begin{array}{l}\text { Withdrawals } \\
\text { and drop-outs }\end{array}$ \\
\hline Autret et al (2015), France & 9 & Weak & Weak & Moderate & Weak & $\mathrm{N} / \mathrm{A}$ & Moderate & Strong \\
\hline Bonneterre et al (2013), France & 9 & Weak & Weak & Moderate & Moderate & $\mathrm{N} / \mathrm{A}$ & Weak & Strong \\
\hline de Koning et al (2017), The Netherlands & 12 & Strong & Moderate & Moderate & Strong & $\mathrm{N} / \mathrm{A}$ & Strong & Moderate \\
\hline Hannerz et al (2011), Denmark & 12 & Moderate & Strong & Moderate & Weak & $\mathrm{N} / \mathrm{A}$ & Strong & Strong \\
\hline Hannerz et al (2012), Denmark & 14 & Strong & Strong & Moderate & Strong & $\mathrm{N} / \mathrm{A}$ & Strong & Strong \\
\hline $\begin{array}{l}\text { Johnson (1987), } \\
\text { Great Britain }\end{array}$ & 11 & Moderate & Weak & Moderate & Moderate & $\mathrm{N} / \mathrm{A}$ & Strong & Strong \\
\hline Ntsiea et al (2015), South Africa & 14 & Moderate & Moderate & Strong & Weak & Moderate & Strong & Strong \\
\hline $\begin{array}{l}\text { Saeki et al (1995), } \\
\text { Japan }\end{array}$ & 7 & Weak & Moderate & Moderate & Weak & $\mathrm{N} / \mathrm{A}$ & Weak & Weak \\
\hline Walker et al (2006), USA & 12 & Strong & Moderate & Moderate & Strong & $\mathrm{N} / \mathrm{A}$ & Strong & Moderate \\
\hline
\end{tabular}

*Total score computed by converting strong $=3$, moderate $=2$, weak $=1$ and $\mathrm{N} / \mathrm{A}=0$, and computing a sum score. Global rating was assigned according to the EPHPP guidelines (strong=no weak ratings, moderate $=$ one weak rating, weak=two or more weak ratings).

$\mathrm{N} / \mathrm{A}$, not applicable.

\section{Quality assessment}

Most studies $(n=8)$ adjusted for potential confounders and had an acceptable response rate of $60 \%$ or more at baseline $(n=9)$. As seen in table 3 , only one-third of the studies $(n=4)$ were rated as strong on the global rating of quality (using EPHPP) ${ }^{38-4047}$; five studies were rated moderate ${ }^{3641-4346}$; and four studies were rated weak. ${ }^{35} 374445$

\section{Evidence synthesis}

In line with the best evidence synthesis guideline, we found that the level of evidence was mostly insufficient, since most specific work factors were only examined in one study (see Evidence synthesis in the Methods section). Only single studies rated as strong are described here since they yield limited evidence on their own (single moderate studies yield insufficient evidence). Studies rated as moderate are only described when they provide evidence in combination with strong studies.

de Koning et $a l^{38}$ was rated as strong and assessed workload in hours and complete RTW (same number of hours 6 and 12 months after mild TBI). The strong study found that workload in hours per week, which formed a nonlinear U-shaped effect, was predictive for 6-month complete RTW following mild TBI. The curvilinear relationship designates less than 25 work hours a week to the highest odds of complete work retention, 32 hours a week as the lowest odds, followed by increased odds with increased workload. The employees who worked less than 25 hours and more than 32 hours were more likely to fully retain work 6 months after mild TBI. Six months later, workload was not predictive for complete RTW in the same study. According to the systematic review evidence synthesis guide, we found limited evidence that workload was unrelated to complete work retention (in terms of the same number of work hours as preinjury) 1 year after ABI. Six months after ABI, we found limited evidence that workload was related to complete work retention in a U-shaped curve. ${ }^{38}$
Among eight studies assessing occupational role and work retention, six studies were rated as strong or moderate (equally divided). 363839414647 Although the studies used different definitions of occupational role (see table 2), all categorised manual (or blue-collar) work as having less educational requirements than non-manual (white-collar, skilled or professional) comparisons. Among these studies, four had straightforward findings that manual workers were less likely to RTW than non-manual workers. $^{36} 414647$ Walker and colleagues' strong study ${ }^{47}$ and Hannerz et al's moderate study ${ }^{41}$ found higher odds of work retention with increased educational requirements of the job. Two strong studies contained findings pointing in different directions. $^{38} 39$ De Koning et al's strong study also found that manual work reduced the probability of full RTW 6 months after mild TBI, compared with skilled work, an effect that was no longer present 1 year after the injury. ${ }^{38}$ However, professional workers (higher than skilled work in educational requirement) also reduced the probability of full RTW, compared with skilled workers. ${ }^{38}$ Endo et al's strong study found that non-manual (desk) workers resigned faster after stroke than manual workers, measured from the first day of sick leave to 1 year after. ${ }^{39}$ In the same study, another occupational role was investigated: managers returned to work faster than non-managers during the same period. Thus, in the four studies ${ }^{36414647}$ (one strong and the remaining moderate) that assessed RTW (as opposed to 'time to resignation' or complete RTW), we found moderate evidence that manual work, compared with non-manual work, increased the likelihood of work retention after ABI. We also found limited evidence (one strong study) ${ }^{39}$ that managers, compared with nonmanagers, were more likely to retain work after $A B I$.

Two strong studies assessed enterprise size and work retention. ${ }^{39}{ }^{40}$ However, the studies defined enterprise size so differently that they were evaluated as single studies. One study reported that large enterprise size ( $\geq 250$ employees) was positively related to RTW 2 years after injury. Each increase in enterprise size (micro, 1-9 employees; small, 10-49 employees; 
medium, 50-249 employees; and large, $\geq 250$ employees) was followed by an increase in the odds of work retention, 2 years after stroke. In contrast, the other study found that enterprise size (dichotomised as $\leq 999$ employees or $\geq 1000$ employees) was unrelated to RTW 1 year after stroke. Thus, we found limited evidence that large enterprise size defined as $\geq 250$ employees is positively related to work retention, while large enterprise size defined as $\geq 1000$ employees was not related to work retention after $A B I$.

\section{DISCUSSION}

This is the first systematic review to comprehensively screen and synthesise evidence of studies examining specific workplace factors and work retention after ABI. Based on 13 included studies, we found moderate evidence of manual work's negative relationship with work retention. We also found limited evidence for (1) a U-shaped relationship between workload and complete RTW at 6 months, and no relationship between workload and complete RTW at 12 months; (2) a manager role, compared with a non-manager role; and RTW; (3) large enterprise size (defined as $\geq 250$ employees) as positively related to RTW; and (4) large enterprise size (defined as $\geq 1000$ employees) as unrelated to RTW.

These findings point towards several necessities and some limitations in the field that should be addressed in future studies. First, onsite workplace adaptions are potential avenues for increased RTW. Examples of work adaptations found in our review are a combination of work trials, assistance from coworkers and adapting work hours, ${ }^{42}$ as well as tailoring workplace interventions according to functional ability and workplace challenges. ${ }^{43}$ Still, there is a lack of a robust evidence base concerning the association between these modifiable workplace factors and work retention after experiencing ABI. Illustrating this, we found that the relationship between work hours and RTW was complex (U-shaped relation), with both employees working less than 25 and more than 32 hours more likely to fully retain work after 6 months, in one study. ${ }^{38}$ Because most specific workplace factors were only assessed in one study; more studies with methodological robust study designs are essential to bring this research front forward.

Second, the under-representation found in our review of manual workers among those who retain work after ABI is supported in new single studies, ${ }^{48}$ as well as studies with other patient groups. ${ }^{50}$ No included studies empirically examined potential mechanisms behind this possible link. ${ }^{51}$ Lower job control and higher job strain, ${ }^{52}$ socioeconomic disadvantage, ${ }^{50}$ premorbid cognitive reserves less conductive to RTW ${ }^{3853}$ and the underdetection of physical impairments (eg, balance problems and fine motor skills) after injury ${ }^{545}$ have been suggested as potential pathways from manual work to reduced RTW. Other possible pathways could be insecure work attachment and less work adaptation by employers for manual compared with nonmanual workers.

The only specific workplace factors examined in more than one original study in this review were descriptive aspects of workplaces, such as occupational role (eg, manual labour vs non-manual labour) and enterprise size. Although these descriptive factors might be informative, there is a clear need to further examine modifiable aspects of work. Until more strong studies among employees with ABI are conducted and replicated, studies on work retention among other patient groups may be useful in indicating modifiable work factors that might be useful after ABI. Access to multidisciplinary resources ${ }^{11}$; better assessment of work capacities, clarity on the rights and obligations of employers and workers alike, and the setup of a positive discrimination employment policy ${ }^{51}$; and job control and (reversed) job strain $^{52}$ are examples of modifiable workplace factors related to work retention found in other patient groups. Our review is thus a reply to the vocational research community's former calls for greater focus on the workplace following many types of injury, not only ABI. ${ }^{11} 12152324515556$ Our findings also demonstrate the need to examine mechanisms and processes at the workplace.

\section{Strengths and limitations of the systematic review}

The current study used a transparent and comprehensive systematic approach in searching, screening and quality assessing the studies according to predefined criteria with at least two independent reviewers. Also, the standardised EPHPP was used to assess study quality, which has been found to have good interrater reliability. ${ }^{34}$ Still, there are some limitations that should be addressed. The few included studies with heterogeneous samples spanning different cultures, patient groups (ie, in type and severity of $\mathrm{ABI}$ ) and different operationalisation of exposure and outcome variables warrant caution in generalising the findings. We would particularly like to note that the review contains studies from a diversity of contexts ranging from industrialised (eg, Japan) to low-income countries, thus most probably reflecting different labour practices and protections across study settings. This limits the generalisability of findings.

Although we are confident that we found most peer-reviewed studies after conducting a comprehensive librarian-assisted search in 11 electronic databases, we did not explicitly search for 'grey literature' (ie, unpublished studies, such as master or doctoral thesis). ${ }^{57}$ This poses a risk of publication bias, although grey literature may have little impact on the results. ${ }^{57}$ Another limitation of the current (and most) review, is that quality assessments are based on the explicit information in the articles and not the actual conduct of the study. This makes it difficult to know the extent of selective reporting (ie, not reporting null findings of some variables).

\section{Implications for practice and research}

Our findings suggest that manual workers might be more in need of work adaptation or vocational rehabilitation services after ABI, which is of importance for employers.Efforts should be taken by employers to understand which types of work adaptations or other intervention efforts employees need to be able to retain work. Although current evidence is mostly limited, possible modifiable efforts might be tailoring work tasks, supplying workplace support and adapting work hours. If possible, employers should team up with researchers to conduct more robust scientific evaluations of such efforts to increase the evidence-based knowledge on the field.

The majority of the included studies used longitudinal designs, adjusted for potential confounders, and mostly used valid and reliable instruments. Still, there are several methodological gaps. Some studies had small sample sizes $(\mathrm{n}<80),{ }^{354245}$ thus restricting statistical power and increasing the risk of false-negative findings (ie, 'type II error'), while others had an unknown response rate (unreported eligible sample). ${ }^{37}$ The majority of the included studies relied on employee data only. Adding a multi-informant perspective, such as employers and vocational support staff, could reduce self-report bias.

In sum, the studies showed large discrepancies in their operationalisation of both the workplace and RTW factor (eg, cut-off in enterprise size). ${ }^{39}$ Aiming at conducting comparable 
operationalisation is important for the possibility to compare across studies and lift the evidence base beyond single studies.

Despite the advantages of RCT designs for minimising selection bias and gaining reliable knowledge to be used for policy and practice, only one of the included studies used an RCT design. ${ }^{43}$ Still, practice-based evidence from large longitudinal cohort studies are also needed to define the effective workplace factors related to RTW and the work retention following $\mathrm{ABI},{ }^{58}$ and temporal associations can be built on to design interventions that are tested with RCT designs.

Finally, the studies were mostly conducted in high-income countries proposing caution in generalising as national policies, welfare and social security systems vary considerably, also across high-income countries. The findings are also limited by the inability to account for societal factors such as national welfare provisions and labour market forces known to impact RTW following $\mathrm{ABI}$.

\section{CONCLUSION}

It is highly notable that the vast majority of research on work retention after $\mathrm{ABI}$ almost solely relies on individual and medical factors, ${ }^{13-22}$ while we have access to very little sound evidence regarding the specific workplace factors involved.

This is the first systematic review that maps and evaluates evidence of specific workplace factors associated with work retention after ABI. We find moderate evidence that manual work is associated with lower work retention, but a need to go further and examine potential mechanisms thereof. There is a need to conduct methodologically robust studies of workplace interventions focusing on modifiable workplace factors to increase RTW among employees with ABI. Compared with the quite extensive literature regarding individual and medical factors, only one RCT regarding workplace variables was identified in this review. This demonstrates that the field of workplace adaptations after ABI is still a long way from taking into account the full complexity of factors that contribute to increasing or decreasing the chance of individuals retaining work after ABI. Also, clinical practice should take into account not only that individual and injury-related factors are at play but also that many aspects related to the workplace will be important to address when helping patients return to their previous workplace.

\section{Author affiliations}

Work Research Institute, OsloMet- Oslo Metropolitan University, Oslo, Norway ${ }^{2}$ Institute of Clinical Medicine, Faculty of Medicine, University of Oslo, Oslo, Norway ${ }^{3}$ Center for Habilitation and Rehabilitation Models and Services (CHARM), Institute of Health and Society, Faculty of Medicine, University of Oslo, Oslo, Norway ${ }^{4}$ Department of Physical medicine and Rehabilitation, Oslo University Hospital, Oslo, Norway

${ }^{5}$ Institute of Clinical Medicine, Faculty of Medicine, University of Oslo, Oslo, Norway ${ }^{6}$ Department of Psychology, University of Oslo, Oslo, Norway

${ }^{7}$ Department of Research, Sunnaas Rehabilitation Hospital, Nesoddtangen, Norway ${ }^{8}$ Institute of Social Psychology, Goethe University Frankfurt, Frankfurt, Hessen, Germany

${ }^{9}$ Center for Habilitation and Rehabilitation Models and Services (CHARM), Institute of Health and Society, Faculty of Medicine, University of Oslo, Oslo, Norway

\section{Twitter Wendy Nilsen @wendy_nilsen}

Acknowledgements We thank Vilde Hoff Bernstrøm, who helped with the study design and screening; Pål Klethagen, who helped with the screening; Katrine Ziesler, who helped with the figure; and Bettina Grødem Knutsen, Malene Wølch Gundersen and Ingjerd Legreid Ødemark, who helped with the systematic literature search.

Collaborators Vilde Hoff Bernstrøm, Pål Klethagen, Bettina Grødem Knutsen, Malene Wølch Gundersen and Ingjerd Legreid Ødemark.

Contributors Study concept and design: DEA, WN, VHB, ØS, HE, NA and ML. Screening: DEA, ØS, LF, PK, HE, NA, EIH, ML and VHB. Quality assessment: DEA
SCRF, WN, EIH, LF, NA, ML, HE and ØS. Literature search: BGK, MWG and ILO. Evidence synthesis: DEA, WN and ML. Funding applications: NA, $\varnothing \mathrm{S}, \mathrm{HE}$ and $\mathrm{ML}$. Drafting of the manuscript: DEA, WN, EIH, SCRF, NA, ML, HE, ØSand LF. Critical revision of the manuscript for important intellectual content: all authors. DEA: guarantor.

Funding This research was partly funded by the Research Council of Norway (grant 256 689); the Work Research Institute, OsloMet-Oslo Metropolitan University; and the Department of Research, Sunnaas Rehabilitation Hospital.

Competing interests None declared.

\section{Patient consent for publication Not required.}

Provenance and peer review Not commissioned; externally peer reviewed.

Open access This is an open access article distributed in accordance with the Creative Commons Attribution Non Commercial (CC BY-NC 4.0) license, which permits others to distribute, remix, adapt, build upon this work non-commercially, and license their derivative works on different terms, provided the original work is properly cited, appropriate credit is given, any changes made indicated, and the use is non-commercial. See: http://creativecommons.org/licenses/by-nc/4.0/.

\section{ORCID iD}

Daniele Evelin Alves http://orcid.org/0000-0001-7877-3343

\section{REFERENCES}

1 Giustini A, Pistarini C, Pisoni C. Chapter 34 - Traumatic and nontraumatic brain injury. In: Barnes MP, Good DC, eds. Handb clin Neurol: Elsevier, 2013: 401-9.

2 Zaloshnja E, Miller T, Langlois JA, et al. Prevalence of long-term disability from traumatic brain injury in the civilian population of the United States, 2005. J Head Trauma Rehabil 2008;23:394-400.

3 Thurman DJ, Alverson C, Dunn KA, et al. Traumatic brain injury in the United States: a public health perspective. J Head Trauma Rehabil 1999;14:602-15.

4 Tagliaferri F, Compagnone C, Korsic M, et al. A systematic review of brain injury epidemiology in Europe. Acta Neurochir 2006;148:255-68.

5 Feigin VL, Nichols E, Alam T, et al. Global, regional, and national burden of neurological disorders, 1990-2016: a systematic analysis for the global burden of disease study 2016. Lancet Neurol 2019;18:459-80.

6 Ma VY, Chan L, Carruthers KJ. Incidence, prevalence, costs, and impact on disability of common conditions requiring rehabilitation in the United States: stroke, spinal cord injury, traumatic brain injury, multiple sclerosis, osteoarthritis, rheumatoid arthritis, limb loss, and back pain. Arch Phys Med Rehabil 2014;95:986-95.

7 Rickels E, von Wild K, Wenzlaff P. Head injury in Germany: a population-based prospective study on epidemiology, causes, treatment and outcome of all degrees of head-injury severity in two distinct areas. Brain Injury 2010;24:1491-504.

8 Donker-Cools BHPM, Daams JG, Wind H, et al. Effective return-to-work interventions after acquired brain injury: a systematic review. Brain Inj 2016;30:113-31.

9 Shames J, Treger I, Ring H, et al. Return to work following traumatic brain injury: trends and challenges. Disabil Rehabil 2007;29:1387-95.

10 Schwarz B, Claros-Salinas D, Streibelt M. Meta-Synthesis of qualitative research on facilitators and barriers of return to work after stroke. J Occup Rehabil 2018:28:28-44

11 Cancelliere C, Donovan J, Stochkendahl MJ, et al. Factors affecting return to work after injury or illness: best evidence synthesis of systematic reviews. Chiropr Man Therap 2016;24:32

12 Cronin S, Curran J, lantorno J, et al. Work capacity assessment and return to work: a scoping review. Work 2013;44:37-55.

13 Crooks CY, Zumsteg JM, Bell KR. Traumatic brain injury: a review of practice management and recent advances. Phys Med Rehabil Clin N Am 2007;18:681-710.

14 Donker-Cools BHPM, Wind H, Frings-Dresen MHW. Prognostic factors of return to work after traumatic or non-traumatic acquired brain injury. Disabil Rehabil 2016:38:733-41.

15 Edwards J, Kapoor A, Swartz RH. Return to work after young stroke: a systematic review. Int J Stroke 2017;12:57.

16 Gagnon C, Lamontagne M-E. Poster 104 effects of length of stay on functional independence in traumatic brain injury: a systematic review. Arch Phys Med Rehabil 2013:94:e47.

17 Gillen G, Nilsen DM, Attridge J, et al. Effectiveness of interventions to improve occupational performance of people with cognitive impairments after stroke: an evidence-based review. Am J Occup Ther 2015;69:6901180040p1-9.

18 Hiekkanen H, Kurki T, Brandstack N, et al. Prognostic factors of return to work after acquired brain injury: a systematic review. Brain Inj 2009;23:396-402.

19 Nightingale EJ, Soo CA, Tate RL. A systematic review of early prognostic factors for return to work after traumatic brain injury. Brain Impair 2007;8:101-42.

20 Ottomanelli L, Lind L. Review of critical factors related to employment after spinal cord injury: implications for research and vocational services. J Spinal Cord Med 2009;32:503-31.

21 van Velzen JM, van Bennekom CAM, Edelaar MJA, et al. Prognostic factors of return to work after acquired brain injury: a systematic review. Brain Inj 2009;23:385-95. 
22 Scaratti C, Leonardi M, Sattin D, et al. Work-Related difficulties in patients with traumatic brain injury: a systematic review on predictors and associated factors. Disabil Rehabil 2017;39:847-55.

23 Krause N, Dasinger LK, Neuhauser F. Modified work and returns to work: a review of the literature. J Occup Rehabil 1998;8:113-39.

24 Ownsworth T, McKenna K. Investigation of factors related to employment outcome following traumatic brain injury: a critical review and conceptual model. Disabil Rehabil 2004;26:765-83.

25 Brasure M, Lamberty GJ, Sayer NA, et al. Participation after multidisciplinary rehabilitation for moderate to severe traumatic brain injury in adults: a systematic review. Arch Phys Med Rehabil 2013;94:1398-420.

26 Graham CA, Yuen KY, Yeung JHH, et al. 379 predicting probability of return to work a four years after moderate and major trauma in Hong Kong: prospective, multi-center, cohort study. Ann Emerg Med 2016;68:S145.

27 Veritas Health Innovation. Covidence systematic review software [program]. Melbourne, Australia, 2018.

28 Alves ED, Enehaug $\mathrm{H}, \varnothing \mathrm{S}$, et al. Role of workplace in job tenure for employees returning to work following acquired brain injury: protocol for a systematic review, 2018. Available: http://www.crd.york.ac.uk/PROSPERO/display_record.php?ID= CRD42018082201

29 Moher D, Shamseer L, Clarke M, et al. Preferred reporting items for systematic review and meta-analysis protocols (PRISMA-P) 2015 statement. Syst Rev 2015;4:1.

30 Schardt C, Adams MB, Owens T, et al. Utilization of the PICO framework to improve searching PubMed for clinical questions. BMC Med Inform Decis Mak 2007;7:16

31 Armijo-Olivo S, Stiles CR, Hagen NA, et al. Assessment of study quality for systematic reviews: a comparison of the Cochrane collaboration risk of bias tool and the effective public health practice project quality assessment tool: methodological research. J Eval Clin Pract 2012;18:12-18.

32 Slavin RE. Best evidence synthesis: an intelligent alternative to meta-analysis. J Clin Epidemiol 1995;48:9-18

33 Breslin FC, Dollack J, Mahood Q, et al. Are new workers at elevated risk for work injury? A systematic review. Occup Environ Med 2019;76:694-701.

34 Irvin E, Van Eerd D, Amick III BC, et al. Introduction to special section: systematic reviews for prevention and management of musculoskeletal disorders. J Occup Rehabil 2010;20:123-6.

35 Autret K, Zouker J, Albanese J-B, et al. Return to work after brain injury: a retrospective study of 85 patients followed by an occupational reintegration unit. Ann Phys Rehabil Med 2015;58:308-11.

36 Bonner B, Pillai R, Sarma PS, et al. Factors predictive of return to work after stroke in patients with mild-moderate disability in India. Eur J Neurol 2016;23:548-53.

37 Bonneterre V, Pérennou D, Trovatello V, et al. Interest of workplace support for returning to work after a traumatic brain injury: a retrospective study. Ann Phys Rehabil Med 2013;56:652-62.

38 de Koning ME, Scheenen ME, van der Horn HJ, et al. Prediction of work resumption and sustainability up to 1 year after mild traumatic brain injury. Neurology 2017;89:1908-14

39 Endo M, Sairenchi T, Kojimahara N, et al. Sickness absence and return to work among Japanese stroke survivors: a 365-day cohort study. BMJ Open 2016;6:e009682.
40 Hannerz H, Ferm L, Poulsen OM, et al. Enterprise size and return to work after stroke. J Occup Rehabil 2012;22:456-61.

41 Hannerz H, Holbæk Pedersen B, Poulsen OM, et al. A nationwide prospective cohort study on return to gainful occupation after stroke in Denmark 1996-2006. BMJ Open 2011;1:e000180.

42 Johnson R. Return to work after severe head injury. Int Disabil Stud 1987:9:49-54.

43 Ntsiea MV, Van Aswegen H, Lord S, et al. The effect of a workplace intervention programme on return to work after stroke: a randomised controlled trial. Physiotherapy 2015;1:eS1108-9.

44 Saeki S, Ogata H, Okubo T, et al. Return to work after stroke. A follow-up study. Stroke 1995;26:399-401.

45 van Dongen $\mathrm{CH}$, Goossens PH, van Zee IE, et al. Short-Term and long-term outcomes of a vocational rehabilitation program for patients with acquired brain injury in the Netherlands. J Occup Rehabil 2017;14:14.

46 Vestling M, Tufvesson B, Iwarsson S. Indicators for return to work after stroke and the importance of work for subjective well-being and life satisfaction. J Rehabil Med 2003;35:127-31.

47 Walker WC, Marwitz JH, Kreutzer JS, et al. Occupational categories and return to work after traumatic brain injury: a multicenter study. Arch Phys Med Rehabil 2006;87:1576-82

48 Aarnio K, RodrГguez-Pardo J, Siegerink B, et al. Return to work after ischemic stroke in young adults: a registry-based follow-up study. Neurology 2018;91:e1909-17.

49 Howe El, Andelic N, Perrin PB, et al. Employment probability trajectories up to 10 years after moderate-to-severe traumatic brain injury. Front Neurol 2018;9.

50 Ervasti J, Vahtera J, Pentti J, et al. Depression-Related work disability: socioeconomic inequalities in onset, duration and recurrence. PLoS One 2013;8:e79855.

51 Kiasuwa Mbengi R, Otter R, Mortelmans K, et al. Barriers and opportunities for returnto-work of cancer survivors: time for action-rapid review and expert consultation. Syst Rev 2016;5:35

52 Gragnano A, Negrini A, Miglioretti M, et al. Common psychosocial factors predicting return to work after common mental disorders, cardiovascular diseases, and cancers: a review of reviews supporting a Cross-Disease approach. J Occup Rehabil 2018;28:215-31.

53 Satz P. Brain reserve capacity on symptom onset after brain injury: a formulation and review of evidence for threshold theory. Neuropsychology 1993;7:273-95.

54 Guskiewicz KM. Assessment of postural stability following sport-related concussion. Curr Sports Med Rep 2003;2:24-30.

55 Williams G, Robertson V, Greenwood K. Measuring high-level mobility after traumatic brain injury. Am J Phys Med Rehabil 2004;83:910-20.

56 Lagerveld SE, Bültmann U, Franche RL, et al. Factors associated with work participation and work functioning in depressed workers: a systematic review. J Occup Rehabil 2010;20:275-92.

57 Hartling L, Featherstone R, Nuspl M, et al. Grey literature in systematic reviews: a cross-sectional study of the contribution of non-English reports, unpublished studies and dissertations to the results of meta-analyses in child-relevant reviews. BMC Med Res Methodol 2017;17:64

58 Horn SD, DeJong G, Ryser DK, et al. Another look at observational studies in rehabilitation research: going beyond the Holy Grail of the randomized controlled trial. Arch Phys Med Rehabil 2005;86:8-15. 